\title{
Rezensionen / recensions / recensioni
}

Zaid, Abdelkarim \& Lebeaume, Joël (Éd.). (2015). La formation d'ingénieurs en alternance, rythmes et temporalités vécues. Villeneuve d'Ascq: Septentrion, Presses universitaires, coll. Éducation et didactiques. 199 p.

L'alternance en formation... un vieux serpent de mer, une évidence dont la mise en œuvre généralisée prend du temps, beaucoup de temps? C'est la question du temps, des temporalités qu'explorent justement les chercheurs ayant contribué à cet ouvrage collectif et cet abord est nouveau. À présent que, en France, la formation d'ingénieurs en alternance sous contrat d'apprentissage, est érigée en voie de professionnalisation et d'insertion professionnelle, et alors que la littérature sur l'alternance abonde notamment dans le champ de la formation depuis quelques décennies - en témoigne la très riche littérature internationale, essentiellement francophone et anglophone, convoquée par les auteurs et qui couvre les 10 pages de la bibliographie en fin d'ouvrage - Zaid \& Lebeaume ont fait le pari d'ouvrir un autre champ d'investigation: celui des rythmes de l'alternance. Car ces rythmes sont multiples: au sein des 54 formations étudiées et proposées dans le chapitre 1 (p.59 pour un tableau de synthèse), «aucun modèle temporel se démarque et la répartition est toujours très éclatée. Le nombre de modèles possibles selon les trois paramètres que sont le profil de l'alternance, la progressivité inter-années du temps global de présence en entreprise et la progressivité inter-années de la durée des périodes en entreprise, reste important. Aucun modèle n'est véritablement prédominant mais six modèles de temporalité se dégagent dont le plus représenté est l'alternance longue, constante, avec allongement» (p.58).

Plusieurs questions orientent la problématique exposée ici: prise en compte du temps dans les recherches sur la formation; rôle de l'histoire des dispositifs, expérience des acteurs dans le choix et la construction des rythmes, rôle de l'écrit dans les mémoires d'apprentis-ingénieurs comme entrée pour analyser les temps vécus et la caractérisation du modèle sous-tendant une formation en alternance donnée. Explicitement, il n'est pas question de mettre en évidence une temporalité idéale. La question est plutôt d'identifier les enjeux culturels, expérientiels et sociaux qui sous-tendent les choix réalisés dans chaque formation et de comprendre comment les acteurs des dispositifs notamment argumentent ou vivent leur temporalité.

Structuré en trois parties, le livre est introduit, sous la plume de A. Zaid et J. Lebeaume par la présentation de la recherche et ses éléments de cadrage. Tous les éléments utiles à une lecture éclairée y sont: Expertise des rythmes de l'alternance; Rythmes, progressivité des apprentissages et production du soi; Principes ayant orienté la recherche; Modalités de recueil et d'analyse de données; la question de l'insensibilité des recherches en éducation à la dimension temporelle; l'état de la 
question sur le temps en formation et en éducation (en formation des ingénieurs mais le temps didactique, les temps sociaux, sa matérialité construite par l'acteur, etc.); Proposition d'un cadre d'analyse du temps en formation en alternance. Cette introduction est fort utile pour comprendre et entrer dans le concept-même des temporalités en formation en général et non seulement en formation d'ingénieurs en alternance.

La première partie propose une entrée par le discours des acteurs sur le temps prescrit et le temps vécu. Abdelkarim Zaid en signe, avec alternativement Catherine Couturier et Joël Lebeaume, les trois chapitres qui portent sur 1) Un panorama de l'alternance dans les formations d'ingénieurs par apprentissage en France: le rythme en question; 2) Les rythmes de l'alternance: ce qu'en disent les apprentis ingénieurs, les tuteurs ingénieurs, les formateurs académiques et les employeurs; 3) dynamiques didactiques en jeu et temporalités - points de vue des apprentis ingénieurs et de leurs tuteurs.

La deuxième partie étudie toujours le temps prescrit et le temps vécu mais, cette fois, en offrant une entrée par les écrits des acteurs. Joël Lebeaume, Dominique Lahanier Reuter, Isabelle Delcambre, Emmanuel Demur et Robert Canonne ont rédigé les trois chapitres qui la composent. 1) Le cadrage prescrit de l'organisation temporelle de l'alternance, au-delà de la question du rythme tente de reconstituer l'ingénierie pédagogique à partir de l'analyse des documents pédagogiques et d'organisation à l'attention des acteurs et de celle du cadrage explicite des temps de l'alternance. 2) Les rapports d'alternance d'apprentis ingénieux sont étudiés dans ce qu'ils ont d'emblématique notamment par rapport aux tensions inhérentes au genre qu'ils révèlent (valoriser une expérience singulière et des compétences génériques; hésiter à dire ses apprentissages et ses réussites; équilibrer descriptions détaillées, contextualisées des apprentissages réalisés et la synthèse de ces derniers), en regard des différentes temporalités. Sont également étudiés les relations aux contenus que les apprentis se sont appropriés, les temporalités indépendantes des contenus et du problème des sources, et enfin les contenus caractérisés par leurs espaces de légitimité. Le dernier chapitre de cette partie revient sur Vingt ans de formation d'ingénieurs par apprentissage, éléments d'histoire et de constats: la nécessité d'un ingénieur nouveau; les spécificités du modèle Ingénieurs 2000; le constat posé au terme de 20 ans de pratique.

La troisième partie propose des éclairages historiques, expérientiels et modélisants sur les dispositifs de formation d'ingénieurs en alternance. Michel Sonntag et David Oget analysent l'articulation entre formation en situation de travail et formation académique dans une formation d'ingénieurs par alternance, en comparant notamment les masters universitaires et spécialisés et en analysant une formation spécifique d'ingénieurs par alternance (spécialité: mécanique). Luc Chevalier étudie une spécificité française: Former des ingénieurs par l'apprentissage à l'ESIPE en partenariat avec le CFA Ingénieurs 2000 (le lecteur trouve, en page 185, un glossaire de toutes les abréviations utilisées). Enfin, en chapitre conclusif, les coordinateurs du livre Joël Lebeaume et Abdelkarim 
Zaid esquissent un essai d'objectivation d'un modèle de l'alternance "explicite et scientifiquement argumenté» avec ses composantes (l'apprentissage, les formes d'articulation entre les deux situations de formation, la construction du contenu enjeu de la formation, la configuration globale du contenu, le tutorat), leurs caractéristiques et principes (p.183). Enfin, des perspectives d'innovation pour une alternance intégrative fonctionnelle sont posées en conclusion. Au cœur de leur principe, les auteurs posent la formation des tuteurs ingénieurs qui «constitue vraisemblablement le levier d'innovation le plus pertinent dans le contexte institutionnel» puisque cette formation permet «de renforcer la professionnalisation et d'intervenir, indirectement, sur les tuteurs enseignants et par effet domino sur les relations partenariales instituées» (p.184).

Relations étroites et paritaires entre terrain professionnel et institution de formation en établissement, telle est la clé d'une alternance digne de ce nom et surtout efficace. Ce n'est certes pas nouveau. Mais le détour par la temporalité, tel que l'ont emprunté les auteurs de cet ouvrage écrit à plusieurs mains, ouvre d'autres manières d'appréhender le processus. Pour le lecteur de la RSSE dont les préoccupations peuvent se situer essentiellement dans le domaine de la formation à l'enseignement, le décentrement sur un autre type formation en alternance, les questions posées et les esquisses de solutions retenues permettent de voir sur un autre angle des problématiques récurrentes et qui demandent encore, clairement, éclaircissements et nouvelles solutions.

Danièle Périsset, Haute école pédagogique et Université de Genève 